\title{
Signatures of Exciton Coupling in Paired
}

\section{Nanoemitters}

\author{
Jack S. Ford, David S. Bradshaw and David L. Andrews* \\ School of Chemistry, University of East Anglia, Norwich Research Park,
}

Norwich NR4 7TJ, U.K.

*Email: d.1.andrews@uea.ac.uk 
ABSTRACT: An exciton formed by the delocalized electronic excitation of paired nanoemitters is interpreted in terms of the electromagnetic emission of the pair, and their mutual coupling with a photodetector. A formulation directly tailored for fluorescence detection is identified, giving results which are strongly dependent on geometry and selection rules. Signature symmetric and antisymmetric combinations are analyzed and their distinctive features identified.

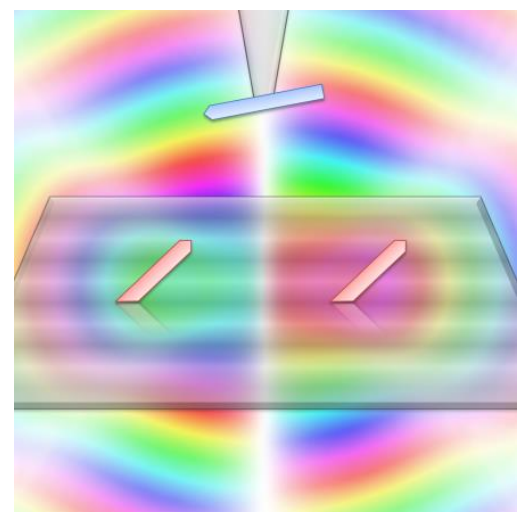

KEYWORDS: fluorescence, nanoantenna, excitons, electronic coupling, nanoparticles, nanophotonics, quantum electrodynamics 


\section{INTRODUCTON}

The concept of optical nanoantennas is based on the nanoscale miniaturization of features that are well-established in radio-frequency technology. ${ }^{1-3}$ Current designs usually exploit the unusual dispersion properties associated with the plasmonic response of metallic nanoparticles at optical frequencies. Recently, attention has turned to shaping the directionality of optical emission through nanoantenna element coupling. ${ }^{4-9}$ Enhancing the efficiency of nanoemitters via interaction with nanoantennas (often composed of gold) is attracting much interest; ${ }^{10-16}$ similarly, the capacity to influence light emission with dielectric nanoantenna arrays is also well recognized. ${ }^{17}$ Along with the burgeoning interest in nanoantennas, attention has now begun to focus on the interplay between fluorescence emitted from closely separated sources. ${ }^{18-20}$ Prominent among the potential applications include solar energy collection, microscopy and sensing. ${ }^{21-23}$

Seeking to exploit the enhanced intensities of emission that can result from nanoantenna interactions, the following analysis addresses particular features arising from nanoemitters in close proximity. In detail, this investigation aims to secure a thorough understanding of the quantum mechanisms operating between a pair of electromagnetically coupled dielectric nanoparticles. Earlier groundwork calculations, ${ }^{24}$ focusing on energy transfer across the pair alongside an emission process, first identified characteristic features in pair fluorescence whose detection we now address. For the first time, rigorous results are presented that will directly predict the signal registered by a photodetector, enabling the process to be fully interpreted. Crucially, these results depend on the positioning of the detector with respect to the location of the emitter pair, and on the orientation of the detector transition dipole moment with respect to the dipole pair responsible for the emission. The explicit coupling of the nanoemitters with the 
detector is included in the theory, leading to a formulation directly tailored for experimental application. The following analysis introduces a construct amenable to the identification of both near- and far-field properties, so that the framework will be applicable not only to a conventional remote detector, but also the near-field microscope setup that is our present focus. Detailed reviews on near-field theory and instrumentation are found in refs 25 and 26.

\section{THEORETICAL METHODS}

Let us consider a system comprising three essential components: two emitters (labeled $A$ and $B$ ) and a photodetector, $D$. The detector is assumed to comprise a suitably absorbing molecular component placed on a support that is movable in the near-field, as for example one held on an AFM tip. Initially $A$ or $B$ occupies an excited state $m$, whilst its neighbor is within its ground state 0 ; detector $D$ is originally in its similarly-labeled ground state. Following the emission process, $A$ and $B$ will be in their ground states, while $D$ is promoted to an excited state $\gamma$ through the act of detection. The energy difference between state $m$ (or $\gamma$ ) and 0 is given by the photon energy of the fluorescent emission, $\hbar c k$.

Detection of the sought optical emission is a result of energy being transmitted from the nanoemitter pair to the photodetector. To properly describe this transmission, we consider both the electromagnetic coupling that operates between each emitter and the detector, and also the coupling between the emitters (involved in producing distinctive features in the pair fluorescence) within a single quantum framework. This is achieved by determining a quantum amplitude, the modulus square of which produces an experimental observable, i.e. the rate of detection. With the excitation localized on $A$, the quantum amplitude (denoted by $M^{A}$ ) is defined as; 


$$
M^{A}=M^{D A}+M^{D A B}+M^{D B A}+M^{B D A}
$$

The leading term of equation (1) signifies direct coupling of $D$ with $A$ - the explicit expression for which is written as; ${ }^{27}$

$$
M^{D A}=\mu_{i}^{\gamma 0(D)} V_{i j}\left(k ; \mathbf{R}_{D A}\right) \mu_{j}^{0 m(A)}
$$

Here, the superscripts on the transition dipoles, $\boldsymbol{\mu}$, designate the nature of the transition, e.g. $\gamma 0$ denotes the transition $\gamma \leftarrow 0$, and $\mathbf{R}_{D A}$ is the displacement of $A$ with respect to $D$; summation over repeated Cartesian indexes is also implied. The other, higher-order, terms of equation (1) which relate to distinct combinations of pairwise coupling (one such arrangement is presented in Figure 1) are highly dependent on the relative positioning of the two nanoemitters. Extending the use of the superscripts on $M$ to represent intermolecular connectivity, we have; ${ }^{28}$

$$
\left.\begin{array}{c}
M^{D A B}=\mu_{i}^{\gamma 0(D)} V_{i j}\left(k ; \mathbf{R}_{D A}\right) \alpha_{j k}^{0 m(A)}(-k ; 0) V_{k l}\left(0 ; \mathbf{R}_{A B}\right) \mu_{l}^{00(B)} \\
M^{D B A}=\mu_{i}^{\gamma 0(D)} V_{i j}\left(k ; \mathbf{R}_{D B}\right) \alpha_{j k}^{00(B)}(-k ; k) V_{k l}\left(k ; \mathbf{R}_{A B}\right) \mu_{l}^{0 m(A)} \\
M^{B D A}=\mu_{i}^{00(B)} V_{i j}\left(0 ; \mathbf{R}_{D B}\right) \alpha_{j k}^{\gamma 0(D)}(k ; 0) V_{k l}\left(k ; \mathbf{R}_{D A}\right) \mu_{l}^{0 m(A)}
\end{array}\right\}
$$

where $V_{i j}(k ; \mathbf{R})$ signifies a electrodynamical coupling tensor that acts between two components, separated by a displacement $\boldsymbol{R}$ of length $R$, serving to define the intermolecular (pairwise) transfer of emission; if zero energy is exchanged, i.e. $k=0$, the coupling is described as static. 
Moreover, the transition polarizability tensor $\boldsymbol{\alpha}^{f i(\xi)}$, given explicitly within ref. 29 , represents the capacity of molecule $\xi$ (i.e. $A, B$ or $D$ depending on the equation) to undergo a two-photon interaction. Generally, all four terms of equation (1) will contribute to the quantum amplitude $M^{A}$, as they connect the same initial and final system states.

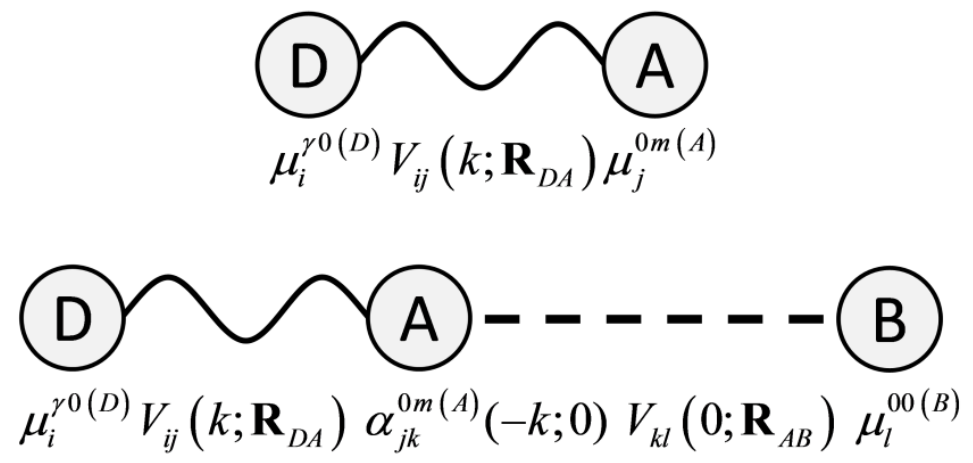

Figure 1. Coupling configurations described by quantum amplitudes $M^{D A}$ and $M^{D A B}$. The coupling between $A$ and $B$ in the latter is static due to $k=0$, illustrated by a dashed line. Two other forms of coupling arise, not depicted here, corresponding to expressions in the second and third lines of equation (3): these are configurations centered on $B$ and $D$.

Since the modulus square of the entire amplitude $M^{A}$ directly relates to the fluorescence detection signal, cross-terms corresponding to quantum interference between the various contributions will also have physical significance. The leading term is provided by the modulus square of equation (2); this is the principal contribution to the detector signal, followed by quantum interference between $M^{D A}$ and the three higher-order amplitudes. Which of the latter contributions is the next-leading term will depend upon their relative magnitudes, as primarily 
determined by the relative displacements of the components - and by the particular selection rules for single-photon and two-photon transitions (associated with transition dipole and transition polarizability interactions, respectively). For example, if $A$ and $B$ are chemically different, and their decay transitions are two-photon and one-photon forbidden, respectively, then $M^{D A B}$ and $M^{B D A}$ will vanish. In such a case, the foremost contribution to the detector signal that is sensitive to the relative positioning of $A$ and $B$ corresponds to $2 \Re \mathrm{Re}\left(M^{D A} M^{D B A}\right)$.

When $A$ and $B$ are identical, significant additional effects arise, since delocalization of the initial excitation across the emitting pair occurs - i.e. an exciton is formed. In these circumstances, the initial quantum state is described by a superposition of two localizedexcitation states, with the appropriate normalization factor included. The relevant combinations, denoted as either symmetric $\left|i^{+}\right\rangle$or antisymmetric $\left|i^{-}\right\rangle$, are defined explicitly by: ${ }^{30}$

$$
\left|i^{ \pm}\right\rangle=2^{-1 / 2}\left|D_{0}\right\rangle\left(\left|A_{m}\right\rangle\left|B_{0}\right\rangle \pm\left|A_{0}\right\rangle\left|B_{m}\right\rangle\right)
$$

where the subscripts denote the status (excited or unexcited) of each molecular state. The nanoemitter pair may undergo fluorescent emission from each of these two exciton states, and the quantum amplitude for the detection of either process is given by;

$$
M_{ \pm}=2^{-1 / 2}\left(M^{A} \pm M^{B}\right)
$$

Each superscript on the right designates the effective position of the localized excitation. Moreover, $M^{A}$ is provided by equation (1), and the chemical similarity implies that the expression for $M^{B}$ takes exactly the same form, with the labels $A$ and $B$ interchanged. 
The character of the detected signal then depends on the capability of resolving two components in the exciton state. Generally, the coupling of the two emitter states will lead to degeneracy splitting of magnitude $2 \mu_{i}^{0 m(A)} V_{i j}\left(k ; \mathbf{R}_{A B}\right) \mu_{j}^{0 m(B)}$. If the magnitude of this coupling is small, and the emission lines in consequence overlap, the detected emission rate will be proportional to $\left(\left|M_{+}\right|^{2}+\left|M_{-}\right|^{2}\right)$ - reducing to the summed result for the independent emitters, i.e. $\left(\left|M^{A}\right|^{2}+\left|M^{B}\right|^{2}\right)$. However, a more interesting case is where the detector is capable of resolving the doublet formed by the symmetric and antisymmetric emission lines. In this instance the associated signals is determined by $\left|M_{+}\right|^{2}$ and $\left|M_{-}\right|^{2}$ respectively. This leads to the possibility of identifying novel features, which is our focus below.

\section{RESULTS AND DISCUSSION}

The present analysis enables us to identify the variation in detected signal as a function of the detector position. For illustrative purposes, the electric field ${ }^{26,27}$ created from the twin nanoemitters can be identified with the result generated by excluding $\mu^{\gamma 0(D)}$ from the above quantum amplitude expressions. A representation of the distribution in optical phase is then determined from the arctangent of the imaginary part, divided by the real part, of the electric field. ${ }^{31}$ Figure 2 shows plots of the fluorescence signal measured by the detector in the near-field, colored by a representation of the optical phase distribution in the detected field. As shown by Figure 2, there are striking differences in the behavior exhibited by each exciton. Although both $M_{+}$and $M_{-}$are equal combinations of the two localized-excitation fluorescence amplitudes $M^{A}$ and $M^{B}$, the difference of sign in their combination is equivalent to the introduction of a $\pi$-phase difference between the two emission components, i.e. 
$M_{-}=2^{-1 / 2}\left(M^{A}+e^{i \pi} M^{B}\right)$. The colors of the brightest regions in Figure 2, nearest $A$ and $B$, exhibit the phases of $M^{A}$ and $M^{B}$, as each localized-excitation component dominates when $D$ is so close to one of the emitters. An interesting consequence of positioning $D$ equidistant from $A$ and $B$ is that $M_{-}$vanishes. This is clearly seen in the lower image of Figure 2; note the nodal plane halfway between $A$ and $B$, which visibly distinguishes the antisymmetric exciton from the symmetric case.
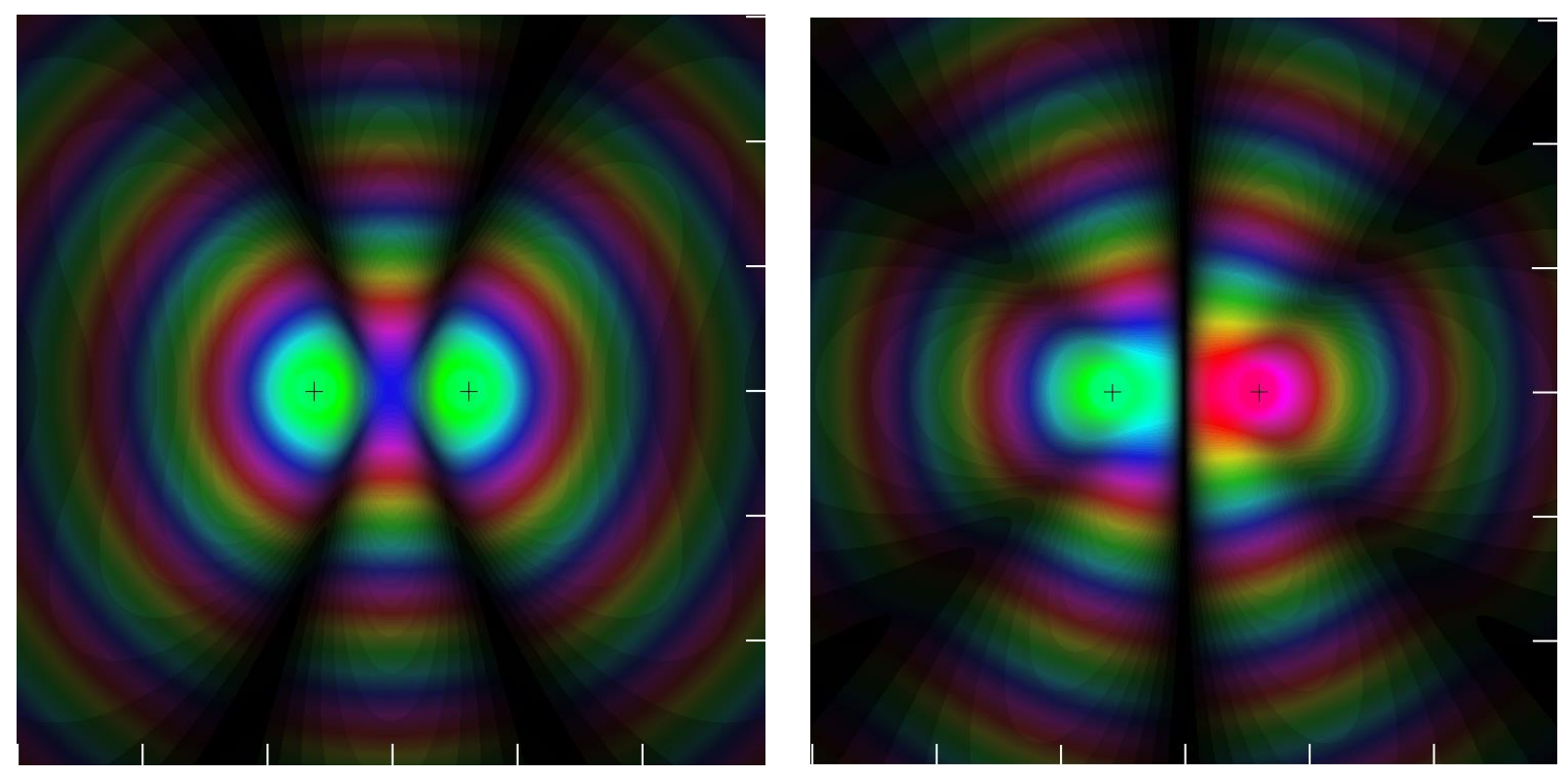

Figure 2: Modulus (brightness) and complex argument (color hue) of the optical fields emitted by: symmetric exciton (left); antisymmetric exciton (right), for near-field positions of the detector $D$. The transition dipoles of $A$ and $B$ are oriented perpendicular to the exhibited plane, and the detector dipole is rotationally averaged, to obviate orientation dependence. The nanoemitters are centered on the cross-marks: the length scale is in units of one wavelength. 


\section{CONCLUSIONS}

A number of interesting conclusions can be drawn from this analysis. It has been shown that excitonic fluorescence emitted by an electronically coupled pair of nanoemitters produces a highly characteristic signal. By specific inclusion of the emission detector in the calculations, our results are directly tailored for practical application. In cases where excitation is localized on a single nanoemitter, coupling with a nearby counterpart produces fluorescence with a distinctive dependence on the relative position and orientation of the pair, and on the static polarizability of each emitter. Conversely, when the initial excitation is delocalized across the pair, the emission conveys signature features characteristic of an exciton doublet. Maps of the detailed intensity and phase distribution reveal striking departures from the character of single-center emission. ${ }^{31}$ For example, using a movable detector, studies of the emission should readily identify a significant dip in detector signal at positions equidistant from the two emitters, in the antisymmetric exciton case. It is interesting to draw a parallel with experimental studies involving the same coherent dipole-dipole coupling that we describe. ${ }^{32}$ In that research, under intense laser irradiation both $A$ and $B$ are excited, rather than merely one of the pair.

In future work we plan to adapt our quantum formulation to address such a case, again seeking to elicit distinctive properties of the ensuing radiation. Continuing to pave the way for experimental studies, a more comprehensive analysis of the collective fluorescence from arrays comprising multiple emitters - including chemical differences, far-field behavior and multiphoton effects - will also appear in upcoming work. 


\section{ACKNOWLEDGMENTS}

The authors are thankful to Dr. Kamel Saadi and Matt Coles for the production of Figure 2. We are also grateful to the Leverhulme Trust and the EPSRC for funding this research.

\section{Notes}

The authors declare no competing financial interest.

\section{REFERENCES}

(1) Novotny, L. Effective Wavelength Scaling for Optical Antennas. Phys. Rev. Lett. 2007, 98, 266802.

(2) Bharadwaj, P.; Deutsch, B.; Novotny, L. Optical Antennas. Adv. Opt. Photon. 2009, 1, 438-483.

(3) Biagioni, P.; Huang, J. S.; Hecht, B. Nanoantennas for Visible and Infrared Radiation. Rep. Prog. Phys. 2012, 75, 024402.

(4) Curto, A. G.; Volpe, G.; Taminiau, T. H.; Kreuzer, M. P.; Quidant, R.; van Hulst, N. F. Unidirectional Emission of a Quantum Dot Coupled to a Nanoantenna. Science 2010, 329, 930-933.

(5) Devilez, A.; Stout, B.; Bonod, N. Compact Metallo-Dielectric Optical Antenna for Ultra Directional and Enhanced Radiative Emission. ACS Nano 2010, 4, 3390-3396.

(6) Lee, K. G.; Chen, X. W.; Eghlidi, H.; Kukura, P.; Lettow, R.; Renn, A.; Sandoghdar, V.; Gotzinger, S. A Planar Dielectric Antenna for Directional Single-Photon Emission and near-Unity Collection Efficiency. Nat. Photonics 2011, 5, 166-169. 
(7) Aouani, H.; Mahboub, O.; Devaux, E.; Rigneault, H.; Ebbesen, T. W.; Wenger, J.

Plasmonic Antennas for Directional Sorting of Fluorescence Emission. Nano Lett. 2011, $11,2400-2406$.

(8) Shegai, T.; Chen, S.; Miljkovic, V. D.; Zengin, G.; Johansson, P.; Kall, M. A Bimetallic Nanoantenna for Directional Colour Routing. Nat. Commun. 2011, 2, 481.

(9) Rolly, B.; Stout, B.; Bonod, N. Boosting the Directivity of Optical Antennas with Magnetic and Electric Dipolar Resonant Particles. Opt. Express 2012, 20, 20376-20386.

(10) Kühn, S.; Håkanson, U.; Rogobete, L.; Sandoghdar, V. Enhancement of Single-Molecule Fluorescence Using a Gold Nanoparticle as an Optical Nanoantenna. Phys. Rev. Lett. 2006, 97, 017402.

(11) Ringler, M.; Schwemer, A.; Wunderlich, M.; Nichtl, A.; Kürzinger, K.; Klar, T. A.; Feldmann, J. Shaping Emission Spectra of Fluorescent Molecules with Single Plasmonic Nanoresonators. Phys. Rev. Lett. 2008, 100, 203002.

(12) Bakker, R. M.; Drachev, V. P.; Liu, Z.; Yuan, H.-K.; Pedersen, R. H.; Boltasseva, A.; Chen, J.; Irudayaraj, J.; Kildishev, A. V.; Shalaev, V. M. Nanoantenna Array-Induced Fluorescence Enhancement and Reduced Lifetimes. New J. Phys. 2008, 10, 125022.

(13) Vecchi, G.; Giannini, V.; Gómez Rivas, J. Shaping the Fluorescent Emission by Lattice Resonances in Plasmonic Crystals of Nanoantennas. Phys. Rev. Lett. 2009, 102, 146807.

(14) Mohammadi, A.; Kaminski, F.; Sandoghdar, V.; Agio, M. Fluorescence Enhancement with the Optical (Bi-)Conical Antenna. J. Phys. Chem. C 2010, 114, 7372-7377.

(15) Agio, M. Optical Antennas as Nanoscale Resonators. Nanoscale 2012, 4, 692-706. 
(16) Lee, K. G.; Eghlidi, H.; Chen, X. W.; Renn, A.; Götzinger, S.; Sandoghdar, V. Spontaneous Emission Enhancement of a Single Molecule by a Double-Sphere Nanoantenna across an Interface. Opt. Express 2012, 20, 23331-23338.

(17) Pellegrini, G.; Mattei, G.; Mazzoldi, P. Light Extraction with Dielectric Nanoantenna Arrays. ACS Nano 2009, 3, 2715-2721.

(18) Evers, J.; Kiffner, M.; Macovei, M.; Keitel, C. H. Geometry-Dependent Dynamics of Two Lambda-Type Atoms Via Vacuum-Induced Coherences. Phys. Rev. A 2006, 73, 023804.

(19) Schmid, S. I.; Evers, J. Dipole-Dipole Interaction between Orthogonal Dipole Moments in Time-Dependent Geometries. Phys. Rev. A 2008, 77, 013822.

(20) Schmid, S. I.; Evers, J. Interplay of Vacuum-Mediated Inter- and Intra-Atomic Couplings in a Pair of Atoms. Phys. Rev. A 2010, 81, 063805.

(21) Corkish, R.; Green, M. A.; Puzzer, T. Solar Energy Collection by Antennas. Solar Energy 2002, 73, 395-401.

(22) Ward, D. R.; Hüser, F.; Pauly, F.; Cuevas, J. C.; Natelson, D. Optical Rectification and Field Enhancement in a Plasmonic Nanogap. Nat. Nanotechnol. 2010, 5, 732-736.

(23) Novotny, L.; van Hulst, N. Antennas for Light. Nat. Photonics 2011, 5, 83-90.

(24) Rice, E. M.; Andrews, D. L. Optical Emission of a Molecular Nanoantenna Pair. J. Chem. Phys. 2012, 136, 244503.

(25) Girard, C.; Dereux, A. Near-Field Optics Theories. Rep. Prog. Phys. 1996, 59, 657.

(26) Girard, C. Near Fields in Nanostructures. Rep. Prog. Phys. 2005, 68, 1883.

(27) Andrews, D. L.; Bradshaw, D. S. Virtual Photons, Dipole Fields and Energy Transfer: A Quantum Electrodynamical Approach. Eur. J. Phys. 2004, 25, 845-858. 
(28) Daniels, G. J.; Andrews, D. L. The Electronic Influence of a Third Body on Resonance Energy Transfer. J. Chem. Phys. 2002, 117, 6882-6893.

(29) Craig, D. P.; Thirunamachandran, T. Molecular Quantum Electrodynamics: An Introduction to Radiation-Molecule Interactions; Dover Publications: Mineola, NY, 1998, p. 113.

(30) Kasha, M.; Rawls, H. R.; El-Bayoumi, M. A. The Exciton Model in Molecular Spectroscopy. Pure Appl. Chem. 1965, 11, 371-392.

(31) Rice, E. M.; Bradshaw, D. S.; Saadi, K.; Andrews, D. L. Identifying the Development in Phase and Amplitude of Dipole and Multipole Radiation. Eur. J. Phys. 2012, 33, 345358.

(32) Hettich, C.; Schmitt, C.; Zitzmann, J.; Kühn, S.; Gerhardt, I.; Sandoghdar, V. Nanometer Resolution and Coherent Optical Dipole Coupling of Two Individual Molecules. Science 2002, 298, 385-389. 